\title{
Reduziert das unterstützte Asthma-Selbstmanagement Exazerbationen?
}

\author{
Für die verschiedensten Erkrankungen liegen zahlreiche \\ Programme für Patientenschulungen vor. Bei Patienten mit \\ Asthma war das frühe Selbstmanagement bei drohenden \\ Exazerbationen effektiv. C. E. Bucknall et al. haben nun geprüft, \\ ob die Kombination aus Selbstmanagement und intensivierter \\ individueller Betreuung bei chronisch obstruktiver Lungen- \\ erkrankung (COPD) Krankenhausaufenthalte reduziert. \\ BMJ 2012; 344: e1060
}

Der präventive Nutzen des aufwendigen Behandlungskonzeptes war gering. Schon die Rekrutierung möglicher Patienten war schwierig. Patienten mit COPD wurden im Krankenhaus angesprochen oder nach einem Aufenthalt angerufen und über das Projekt informiert. Nur die Hälfte war mit einer Teilnahme einverstanden.

\section{Geringes Interesse an einer Teilnahme \\ $\nabla$}

Insgesamt erhielten 464 Patienten eine intensivierte oder eine Standardbetreuung. Alle Patienten optimierten Inhalationstechniken, wurden zum Rauchverzicht aufgefordert und nahmen teilweise Rehabilitationsmaßnahmen in Anspruch. Sie führten Patiententagebücher, beantworteten Fragebögen und wurden jeden Monat nach Exazerbationen befragt. Bei der konventionellen Vorgehensweise erfolgte die Betreuung durch den Hausarzt und Krankenhausspezialisten, die ständig für Notfälle erreichbar waren. Die Interventionsgruppe erhielt zuhause eine umfangreiche Schulung zum Selbstmanagement in 4 jeweils 40-minütigen Trainingssitzungen durch spezialisierte Krankenschwestern. Weitere Besuche schlossen sich in 2- und anschließend in 6-wöchigen Abständen an. Nach definierten Kriterien stand den Patienten eine Notfallmedikation für die umgehende Anbehandlung einer Exazerbation zur Verfügung. Primäre Zielvariablen der Auswertung waren erneute Krankenhausaufenthalte nach 12 Monaten.
111 Patienten der Interventionsgruppe (48\%) und 108 Kontrollpersonen (47\%) kamen aufgrund akuter Exazerbationen in die Klinik (Hazard Ratio [HR] 1,05; 95\%-KI 0,80-1,38; p=0,73). 39 Patienten starben krankheitsassoziiert, wobei keine Gruppenunterschiede bestanden. Sekundäre Zielvariablen waren die psychosoziale Gesundheit und Lebensqualität. Nur 57\% der Patienten schickten hierzu die Fragebögen zurück, sodass eine Auswertung nicht zuverlässig möglich war.

In einer Subgruppenanalyse wurden 75 Patienten, die sich als erfolgreiche „Selbstmanager“ einschätzten, mit den übrigen Teilnehmern der Interventionsgruppe verglichen. Sie waren jünger und lebten seltener allein. Krankenhausaufnahmen und Todesfälle im Zusammenhang mit COPD-Exazerbationen waren in dieser Gruppe seltener (27 vs. $49 \%$; $\mathrm{p}=0,003$ ). Die Unterschiede zur Kontrollgruppe waren geringer (HR 0,60; 95 \%-KI 0,36-0,99; $\mathrm{p}=0,045$ ).

\section{Fazit}

Nach Angaben der Autoren hatte die Kombination aus Selbstmanagement und intensivierter individueller Betreuung nur für einen Teil der Patienten mit COPD einen präventiven Effekt. Das unterstützte Selbstmanagement eigne sich demnach nicht als flächendeckende Präventionsmaßnahme. Ein durchschlagender gesundheitlicher oder ökonomischer Erfolg sei damit nicht zu erwarten.

Dr. Susanne Krome, Melle
Tuberkulose

\section{Informationsbericht des DZK erschienen}

Das Deutsche Zentralkomitee zur Bekämpfung der Tuberkulose (DZK) hat im August seinen 35. Informationsbericht 2012 veröffentlicht. Der diesjährige Bericht enthält wieder aktuelle Beiträge zu den wichtigen Problemen, Entwicklungen und Fortschritten hinsichtlich der Bekämpfung der Tuberkulose in Deutschland.

Neben Informationen zur Epidemiologie der Tuberkulose für das Jahr 2010 sowie einer Auflistung der wichtigsten Links als Recherchehilfe bei allen Fragen rund um die Tuberkulose finden Sie in diesem Jahr auch interessante Beiträge zum Vorgehen bei zwangsweiser Absonderung von Tuberkulosepatienten nach dem Infektionsschutzgesetz. Desweiteren enthält der Informationsbericht Beiträge zu Lieferengpässen bei der medikamentösen Patientenversorgung, zur Spezifität eines Vollblut-Interferon-Gamma-Release-Assay (IGRA) bei Auszubildenden im Gesundheitssdienst sowie zu den Kosten und der Kosteneffektivität unterschiedlicher Tuberkulose-Screening-Strategien.

Auch über die Highlights vom 42. Weltkongress der „International Union Against Tuberculosis and Lung Disease“ (IUATLD) im Oktober 2011 in Lille, Frankreich, werden Sie im aktuellen Bericht informiert. Der 35. Informationsbericht 2012 des DZK kann zum Preis von $15 €$ zzgl. Porto und 7\% MwSt beim DZK unter folgender Adresse erworben werden:

Deutsches Zentralkomitee zur

Bekämpfung der Tuberkulose HELIOS Klinikum Emil von Behring Lungenklinik Heckeshorn

Walterhöferstraße 11

14165 Berlin

E-Mail: info@dzk-tuberkulose.de

Mitteilung des DZK, Berlin 